\title{
Reduction of Adult Intussusception: More Benefit than Harm: Reply
}

\author{
Hirotaka Honjo $^{1} \cdot$ Makio Mike $^{1} \cdot$ Nobuyasu Kano $^{1} \cdot$ Hiroshi Kusanagi $^{1}$
}

Published online: 12 June 2015

(C) Société Internationale de Chirurgie 2015

Dear Sir,

Thank you for your feedback regarding our manuscript.

With regard to postoperative complications among our 41 surgeries, there were three cases of superficial surgical site infection (SSI), two cases of postoperative ileus (POI), and one case of transient renal failure. Thirty-four cases included surgical anastomosis, but there were no cases of anastomotic leakage. Among those 34 cases, one case required covering stoma because of intestinal edema.

Next, we would like to respond to points (a) and (b).

As we described in the "Discussion" section, careful radiologic or endoscopic evaluation can detect strangulated intussusception that is impossible to reduce preoperatively. In other words, we should not be too aggressive in our efforts to reduce intussusception. We do not mean "we should reduce intussusception preoperatively and intraoperatively," but rather, "we should attempt to reduce intussusception preoperatively and intraoperatively, and should know when to stop our attempts to reduce intussusception." In fact, some of the cases involved emergent surgery, and in 12 cases, it was impossible to reduce intussusception.

When preoperative reduction is successful, this affords time for the intestinal edema to resolve, thereby reducing the need for emergency surgery and reducing the chances of postoperative complications. No studies have reported that successful reduction of intussusception is harmful.

Hirotaka Honjo

h-honjo@wood.odn.ne.jp

1 Department of Surgery, Kameda Medical Center, Kamogawa, Japan 